\title{
Transient cortical blindness after coronary angiography
}

\author{
R Parry, J Russell Rees, P Wilde
}

\begin{abstract}
Transient visual loss lasting three days developed after transfemoral coronary angiography in a 62 year old man. Computed tomography (CT) showed bilateral leakage of contrast medium into the occipital cortex. A repeat CT scan after his sight recovered showed clearance of contrast with no underlying infarction.
\end{abstract}

A breakdown of the blood-brain barrier with direct neurotoxicity of the contrast media seemed to be the cause of these neurological changes after coronary angiography which apparently have not been reported before.

(Br Heart f 1993;70:563-564)

\section{Case report}

A 62 year old man was admitted for routine cardiac catheterisation. He had had ischaemic heart disease for 17 years. Four years earlier he had undergone coronary artery bypass grafting with saphenous vein grafts to his circumflex, obtuse marginal, and posterior descending arteries and a left internal mammary artery graft to his left anterior descending artery. He was pain free for 18 months afterwards but angina gradually returned and now severely restricted his activities. Despite treatment with $\beta$-blockers, calcium antagonists, and long acting nitrates he was using up to 100 glyceryl trinitrate tablets weekly. He took $150 \mathrm{mg}$ of aspirin daily.

In addition to his ischaemic heart disease he had intermittent claudication in both calves. The severity of the claudication became clear after the coronary artery bypass surgery and he had bilateral femoro-popliteal artery grafts. He had a history of long-standing hypertension that predated the development of his ischaemic heart disease. Until the time of his coronary surgery he smoked 30 cigarettes daily.

His coronary angiography was technically difficult. The procedure was performed from the right femoral artery with $6 \mathrm{~F}$ Judkins catheters introduced through a haemostatic sheath. A left ventriculogram and an aortogram were performed. One patent vein graft was identified and selectively catheterised. Two vein graft stumps were found. The internal mammary artery proved difficult to engage selectively because of the tortuosity of the proximal subclavian artery and the unusual angle of takeoff of the internal mammary artery from the subclavian artery. The total procedure required $270 \mathrm{ml}$ of iopamidol 370. There were no untoward events during the procedure.

Five minutes after removal of the femoral artery sheath the patient complained that his vision was deteriorating. Over the next $4 \mathrm{~min}$ utes he lost all vision except the ability to distinguish between light and dark. A full neurological examination at this time also showed some loss of coordination in the right arm. There were no other neurological findings and full movement was preserved in all limbs. The presumptive diagnosis was of an embolus to the posterior cerebral ciculation. Computed tomography (CT) was performed to exclude an intracerebral bleed and to establish a baseline in the event of further deterioration in his symptoms. The CT scan (fig 1) obtained 2 hours after the angiogram showed considerable contrast enhancement of both occipital lobes. There was no evidence of any intracerebral bleed or recent infarction.

Over the next three days the patient's sight gradually returned until, by the third day, no visual deficit could be detected on formal testing. The loss of coordination affecting the right arm had also improved but had still not completely resolved by this time. A repeat CT scan on day 3 (fig 2) showed complete clearance of contrast from the occipital lobes with no evidence of underlying infarction in this area.

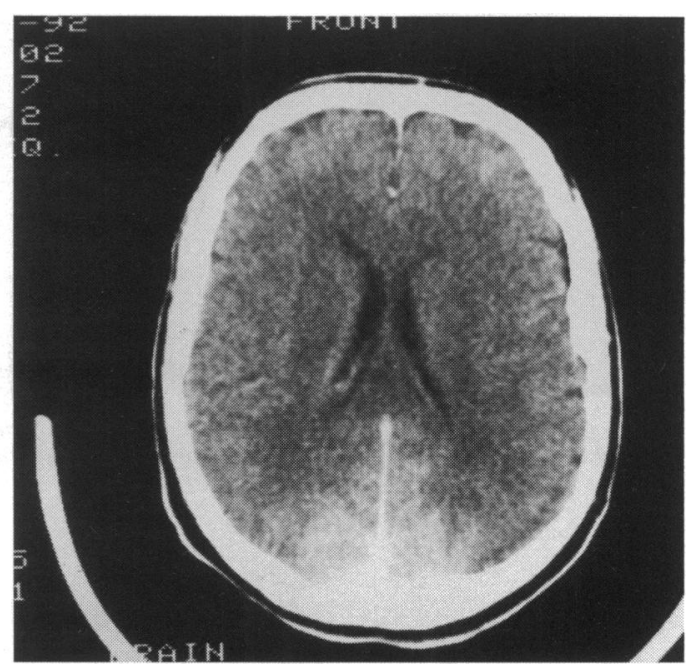

Figure 1 CT scan showing considerable contrast enhancement of the occipital lobes $2 h$ after the angiogram. 


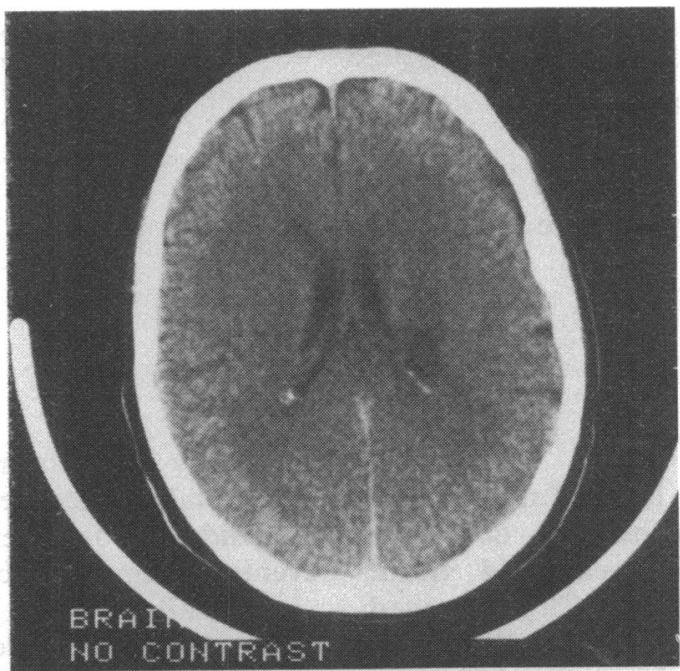

Figure $2 C T$ scan 3 days after the angiogram showing complete clearance of contrast from the occipital lobes with no evidence of underlying infarction.

\section{Discussion}

Non-ionic contrast agents became generally available in 1982. Animal experiments had already shown them to be less damaging to the blood-brain barrier when injected into the cerebral circulation than the ionic contrast agents then in use. Clinical studies have not demonstrated any real advantage from this property and morbidity from neurological sequelae seems to be the same with ionic and non-ionic contrast media. ${ }^{1}$

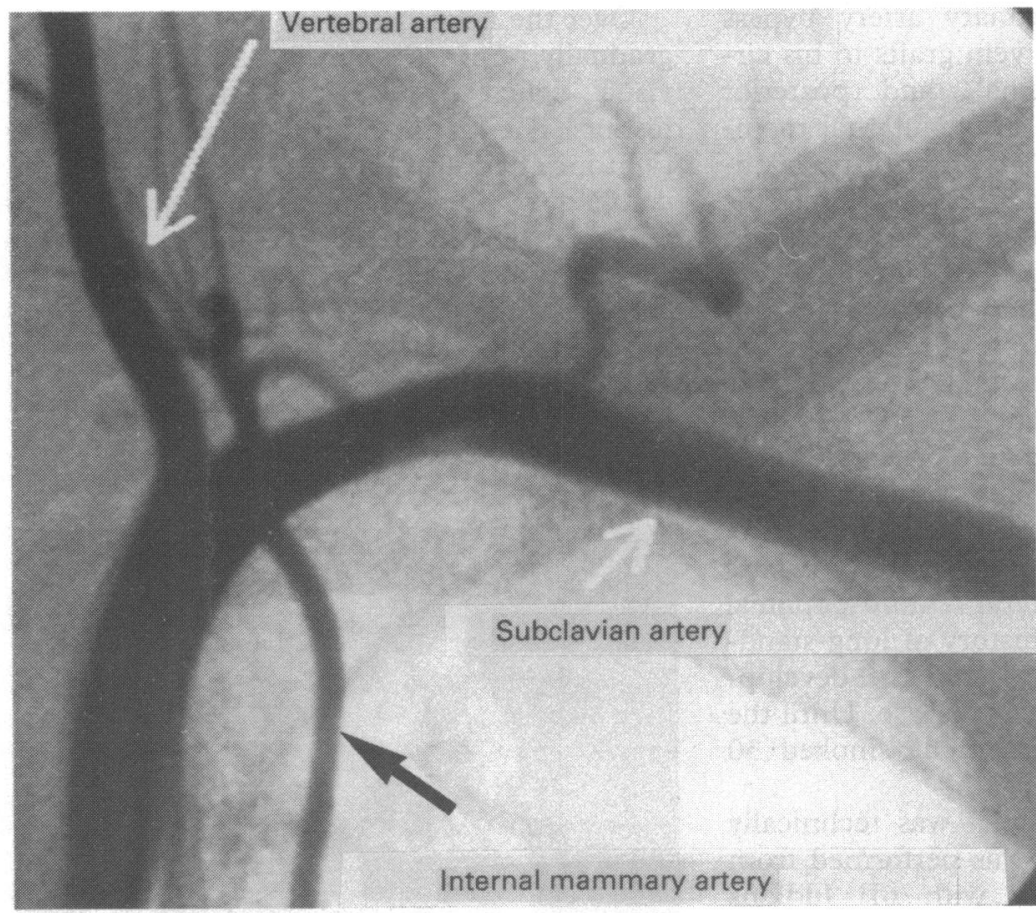

Figure 3 Selective subclavian angiography showing the proximity of the origins of the internal mammary and vertebral arteries from the subclavian artery.
Transient cortical blindness during angiography has previously been assumed to be caused by embolisation or other factors impairing cortical perfusion. Disruption of the blood-brain barrier induced by contrast medium with direct neurotoxicity has been proposed as a potential mechanism. This may be especially true of patients with widespread atherosclerotic disease as in this case.

There have been previous reports of bloodbrain barrier leakage demonstrated by CT (including one case of transient cortical blindness) ${ }^{2}$ in patients who had acute neurological sequelae after selective cerebral angiography. In our patient CT showed selective breakdown of the blood-brain barrier in the posterior cerebral circulation that must have allowed contrast medium to permeate into the occipital cortex. The suggested disruption of the blood-brain barrier is supported by the CT appearance of contrast in the occipital cortex which completely cleared within 3 days with no evidence of underlying infarction. It is difficult to explain why disruption of the blood-brain barrier was confined to the occipital lobes. Studies of cerebral angiography showed that the neurological complication rate increases with the amount of contrast media used. ${ }^{3}$ The internal mammary artery and the vertebral artery arise in close proximity from the subclavian artery (fig 3 ) and it is possible that when the left internal mammary graft was sought a large amount of contrast travelled by way of the left vertebral artery to the posterior cerebral circulation.

Other factors that can contribute to the leakage of contrast are the supine position of the patient and the density of the contrast media compared with blood. While both these may combine to delay clearance of contrast from the occipital lobes they would have no greater effect in this case than in any prolonged angiographic procedure, particularly selective vertebral angiography-in which considerable amounts of contrast are used in the region of the vertebral artery.

Neurological sequelae after angiography may not be related only to embolic events as previously assumed. When a CT scan shows evidence of disruption of the blood-brain barrier with contrast leakage into the cerebral cortex, it may be reasonable to expect that the neurological disturbance will resolve.

1 Cronqvist S. Iohexol in cerebral angiography. Survey and current state. Acta Radiol Suppl 1983;366:135-9.

2 Shyn PB, Bell KA. Transient cortical blindness following cerebral angiography. $f \mathrm{La}$ State Med Soc 1989; 141 (supp ii):35-7.

3 Leow K, Murie JA. Cerebral angiography for cerebrovascular disease: the risks. Br f Surg 1988;75:428-30. 